\title{
Synthesis of Asynchronous Controllers on FPGA from Generalized Multi-Burst Graph Specification
}

\author{
Duarte L. Oliveira $^{1}{ }^{1 *}$, Luiz S. Ferreira ${ }^{1}$, Leonardo Romano ${ }^{2}$ \\ ${ }^{1}$ Divisão de En genharia Eletrônica do Instituto Tecnológico de Aeronáutica - ITA - São José dos Campos - SP, Brazil \\ ${ }^{2}$ Departamento de Engenharia Elétrica do Centro Universitário da FEI - São Bernardo dos Campos - SP, Brazil
}

\begin{abstract}
On promising area application of asynchronous is in Heterogeneous Systems (synchronous and asynchronous modules mixed). Asynchronous controllers are quite used in heterogeneous systems. The specification of theses controllers requires two types of signals: level sensitive signals that are used as conditionals and transition sensitive signals. Another requirement is to describe concurrency between inputs/outputs. The Multi-Burst Graph (MBG) specification allows to describing these controllers in a compact form and it is familiar to designers of digital circuits. This paper proposes a generalization in the MBG specification to increase the ability to describe the interaction between inputs/ outputs, i.e. increase the concurrency between them. Th is paper also proposes a method that starts from Generalized $M B G$ specification and implements its hazard-free controllers on FPGAs. These devices have been mainly used for design of synchronous controllers. However, it is difficult to design asynchronous controllers on FPGAs, because the circuit may suffer from hazard problems. The method proposed implements this class of asynchronous controllers on FPGAs which are based on Look-Up Table (LUT) architectures. By doing this, the asynchronous circuits besides their intrinsic advantages over synchronous ones may also take advantage of integration, lower costs and short-time design associated with FPGA designs.
\end{abstract}

Keywords Asynchronous Logic, Finite State Machine, FPGA, Hazard, Multi-Burst Graph, Synthesis, XBM Specification

\section{Introduction}

There has been a gro wing interest in asynchronous circuits in recent years due to the increase in performance and complexity of digital systems[1]. Asynchronous circuits present several potential advantages over their synchronous counterparts: dissipate less power, do not present problems of clock skew and clock distributed network, and are more robust in respect to temperature variations and electromagnetic interactions[2]. However it is not easy to design asynchronous circuits free of hazards and critical races [2].

Two promising styles of asynchronous systems design are: micropipe line [3] and deco mposition (controllers+data-paths) [4]. For a set of applications, as intensive control-flow design, the decomposition style is the most appropriate. The main reason is the non-pipelined nature of the applications. For this decomposition style different techniques were proposed to the synthesis of data-paths and controllers[5]. An important class of as ynchronous controllers is one that obeys the delay model, Bounded Gated and Wire Delay (BGWD) [2],[6]. This model is realistic, it allows implementing these circuits with basic gates.

\footnotetext{
* Corresponding author:

duarte@ita.br (Duarte L. Oliveira)

Published online at http://journal.sapub.org/msse

Copyright (C) 2012 Scientific \& Academic Publishing. All Rights Reserved
}

\subsection{Specifications for Asynchronous Controllers}

Two important specifications for BGWD asynchronous controllers are: Signal Transition Graph (STG) and Extended Burst-Mode (XBM).

$\boldsymbol{S T G}$ : proposed by Chu in[7] is a Petri-net description (see Figure 1). The strength of STG is to describe concurrence between inputs and outputs (I/O concurrence) that occur in asynchronous systems. It naturally describes timing diagram that are quite used in the interfaces design. However, there are several descriptions in STG that aren't implemented. The description becomes very confusing when it has to man ipulate a larger nu mber of signals. Furthermo re, this type of description may explode in size and also the STG is not so familiar to designers of the synchronous world[8],[9].

$\boldsymbol{X B M}$ : proposed by Yun and Dill in[10] is an extension of burst-mode (BM) specification proposed by Davis in[11] and forma lized by No wick in[12] (see Figure 2). It is suitable for to describe heterogeneous (synchronous / asynchronous) systems behavior, in the case asynchronous finite state mach ines in Mealy type. The XBM specification solves the problems related to the STG description but the XBM allows describing a limited $\mathrm{I} / \mathrm{O}$ concurrency[1]. The XBM is the natural description of finite state machines ${ }^{1}$. These machines interact with the environment in generalized fundamental

\footnotetext{
${ }^{1}$ The 3D-tool synthesizes XBM controllers[10].
} 
mode (GFM). In this mode, a new set of input signals will be activated only if the controller is in a stable state. The XBM specification added two signal types to the BM specification: directed don't-care and conditionals. The latter are level sensitive signals (LSS), i.e., they are active during their " 0 "or " 1 " phase as opposed to all others that are transition sensitive signals (TSS), i.e., they are active during the " $0 \rightarrow 1$ or $1 \rightarrow 0$ " transition. LSS signals may present non-monotonic behaviour. A signal is either LSS or TSS (it may NOT change from one type to the other). Very good results were presented for XBM controllers like SCSI[13], differential equation solver[14] and instruction decoder[15]. Figure 2 shows the input signal Cntgtl that is LSS and the input signals $O k$, Fain, Rin are TSS.

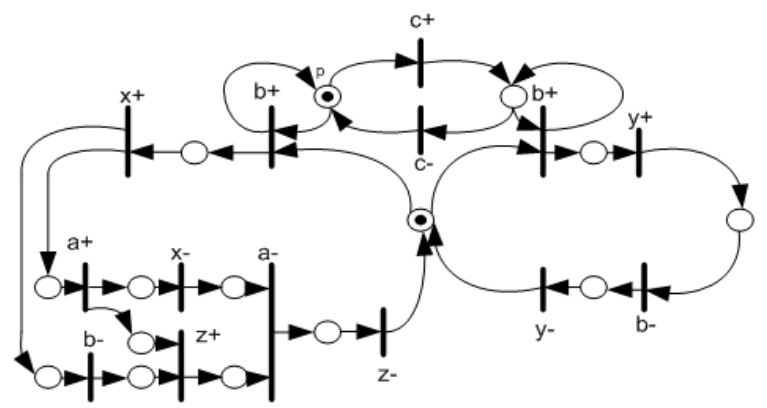

Figure 1. ST G specification in[31]

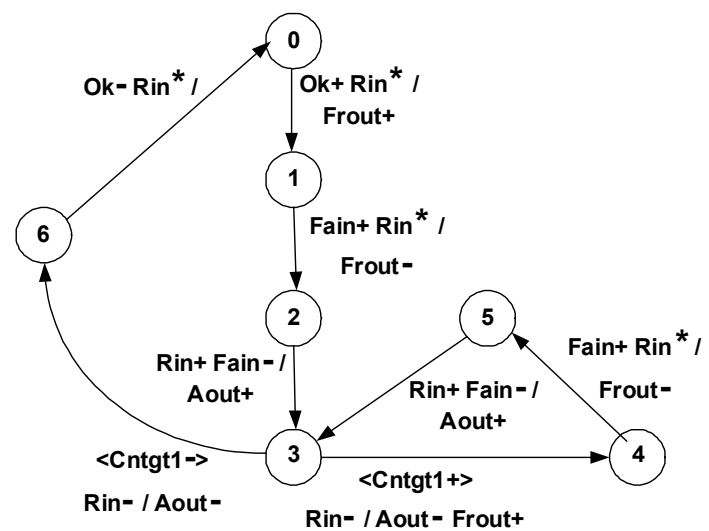

Figure 2. XBM specification: Scsi-init-send

There exist applications whose behaviour contains "four phase signals" (4PS). Such signals may change from TSS to LSS behaviour during the controller's operation[16]. Hence, the signal is active either during its value transition $(0 \rightarrow 1$, $1 \rightarrow 0)$ or during its stable value $(=1,=0)$. Vanbekbergen et al. in[17] proposed the use of 4PS signals targeting their generalized STG. One limitation of their approach is the fact that the STGdescription is later transformed into a SG (state graph) that grows exponentially for large problems (which is the case when non-monotonic LSS signals are present) Recently Kraus et al. in[18],[19] proposed the XBM2PLA tool for XBM machines, starting fro ma more flexible XBM specification (F_XBM) that accepts 4PS signals. He showed that the use of a 4PS signal reduces the size of the circuit's description producing the same result.

Figure 3 shows a flexible extended burst mode specification (F XBM) of the HP Post office benchmark, with 3 inputs (Req, Ackline, Done), 2 outputs (Sendline, Ack) and initial state 0 . The input signal Done is being used in the state transition $3 \rightarrow 0$ by the transition of descent (TSS), while in the state transitions $1 \rightarrow 2$ and $1 \rightarrow 3$ this signal is used by the level value (LSS).

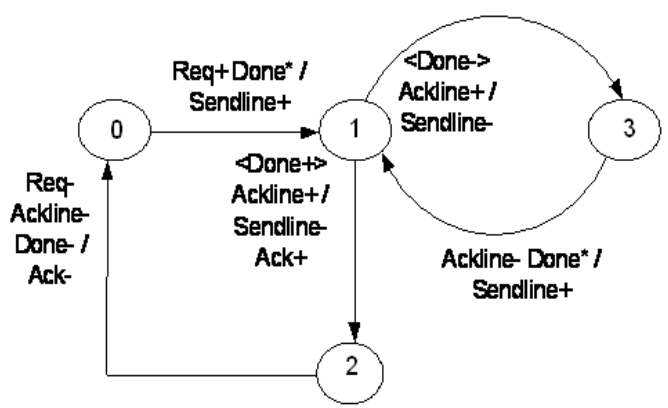

Figure 3. F_XBM specification: HP-Sbuf-Send-Pkt2

Recently, Oliveira et al.[20],[21] proposed the Multi-Burst Graph (MBG) specification. It accepts all signals types of the XBM specification and introduces burst operators. The burst operators allow the description of a limited amount of I/O concurrency. There are three types of operators: input burst OR, transition sequence (SEQ) and transition concurrence $(\mathrm{CO})$. To increase the I/O concurrency it's allo wed to combine the operators CO and SEQ[21]. Oliveira et al.[22] shows that signals 4PS (F_XBM) can be incorporated into the MBG specification. Figure 4 shows a MBG specification using the $\mathrm{CO}$ operator (state transition: 2 $\rightarrow 3$ ). It is the description in MBG of the controller described in STG of Figure 1. The concurrency described in this example can not be captured so much in the XBM specification as also in the F_XBM specification.

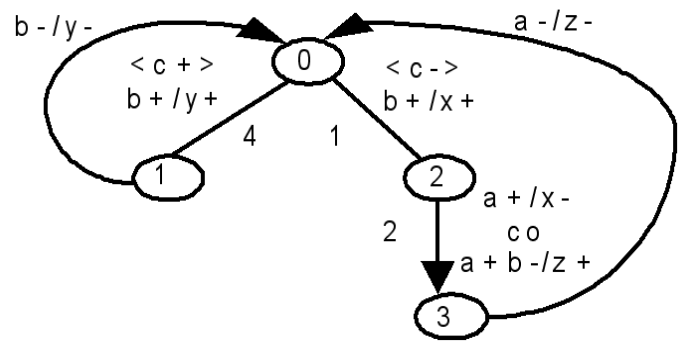

Figure 4. MBG specification

\subsection{FPGA Implementation of B GWD Controllers}

FPGAs are popular components for prototyping and production of digital circuits due to their low cost and short-design time. Their focus has been on synchronous digital circuits. There have been some recent efforts to prototype asynchronous circuits on both commercial[23],[24] and academic FPGAs[25]. There are two reasons as to why off-the-shelf FPGAs are not fit to BGWD controllers [23],[26] and[27]:

a. Mapping Process of hazard-free Booleans functions to logic blocks (macro-cells) may introduce logic hazards. The mapping free of logic hazard, should decomposed gates with fan-in related with LUTs[28]. Each gate is associated a LUT. 
b. Internal Routing Process among logic blocks may introduce significant delays that may result in essential hazard. This type of hazard can be solved by delay elements insertion or if the specification satis fied the requirement of ess ential signal[29].

This paper proposed a generalization of $M B G$ specification, through of extension of SEQ operator. The extension of the SEQ gives a better interaction with the environment in the I/O-Mode, i.e. increases the ability of to describe the I/O concurrency. We also proposed a method that starts from generalized multi-burst graph (GMBG) specification and synthesize asynchronous controllers. They are implemented in the feedback RS standard architecture (see Fig. 5) and interact with the environment in the GFM. This architecture is more robust to ess ential hazard, and the GFM enables the mapping free of logic hazard. The feedback in the RS architecture allows a covering hazard-free logic in the state transitions labelled with signals LSS and that operate with any mach ine cycle (for examp le: Input burst $\rightarrow$ output burst // state variable) ${ }^{2}$. For implement on FPGAs the our GMBG controllers use a more robust logic minimization, where the controllers satisfy the delay model that is unbounded gate delay and bounded wire delay.

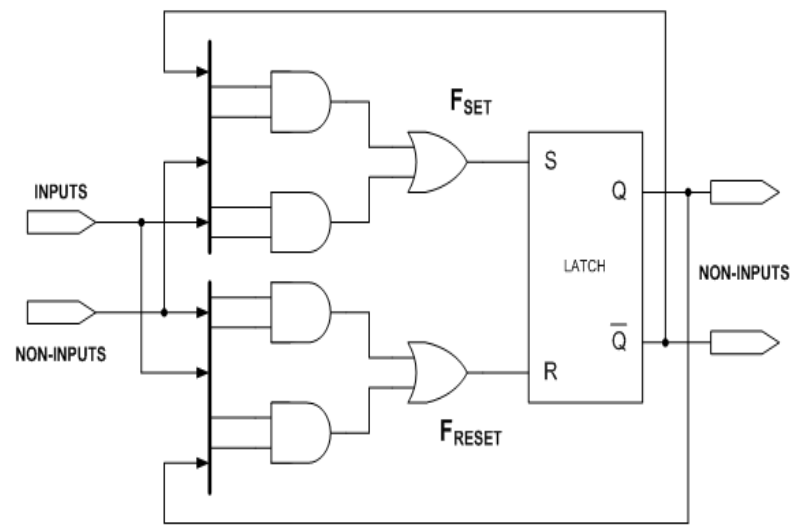

Figure 5. Architecture: feedback RS standard

This paper is structured as follows: section 2 presents the MBG specification, section 3 presents the GMBG specification; section 4 describes the synthesis procedure, section 5 illustrates our method with an example, section 6 shows our experimental results and the section 7 brings the conclusions and future works.

\section{MBG Specification}

The Multi-Burst-Graph (MBG) specification belongs to a class of specifications that allow multiple-input change. The MBG is an extension of the XBM. As in the XBM, MBG is represented as a state graph where each node represents a state and each arc represents a transition. Each transition in the MBG can be activated by: 1) an input burst; or 2) a burst expression. The MBG introduced three operators: input burst $(\mathrm{OR})$, transition concurrence $(\mathrm{CO})$ and transition sequence

\footnotetext{
2 The symbol// means concurren cy.
}

(SEQ). The burst expressions are based on these operators, so it increases the possibilities to describe I/O concurrency.

During the multi-burst transition is assumed that each output signal changes its value just once. If this assumption is not respected a functional hazard may occurs. As in the $\mathrm{XBM}$, in order to guarantee the implementation of an $\mathrm{MBG}$ specification, it must obey restrictions in[20],[21].

Figure 6 shows a MBG specification, the initial state is 0 . The inputs are: $a, b, c$ and $d$. Where $a$ is a level signal type and the others are transition signals type. The outputs are: $x$, $y$ and $z$. In the state transition $5 \rightarrow 6$ is present the OR operator ( $\mathrm{T}_{\mathrm{OR}}$ type). It allow or causality between the signals $b$ and $d$. In the next transition, $6 \rightarrow 7$, the signal $b \sim$ has an undetermined value and the signal $d+$ either is $d=1$ or $\mathrm{d}=0 \rightarrow 1$. In the state transition $2 \rightarrow 3$ the signal $d^{*}$ is directed don't-care. In the state transition $7 \rightarrow 1$ the signal $b+$ either is $b=1$ or $b=0 \rightarrow 1$. The $\mathrm{CO}$ operator is present in the state transition $2 \rightarrow 3$ ( $\mathrm{T}_{\mathrm{CO}}$ type). In the $\mathrm{T}_{\mathrm{CO}}$ type the bursts $\mathrm{b}+/ \mathrm{z}^{+}$ and $\mathrm{c}-\mathrm{d}^{*} / \mathrm{y}+\mathrm{x}+$ are activated concurrently. The SEQ $(>)$ operator is present in state transition $1 \rightarrow 5$ ( $\mathrm{T}_{\mathrm{SEQ}}$ type). The SEQ and CO operators are presents in the state transition $4 \rightarrow 0 \quad\left(\mathrm{~T}_{\mathrm{S}-\mathrm{C}}\right.$ type $)[22]^{3}$. The behaviour of $\mathrm{CO}$ and SEQ operators are detailed in[20],[21].

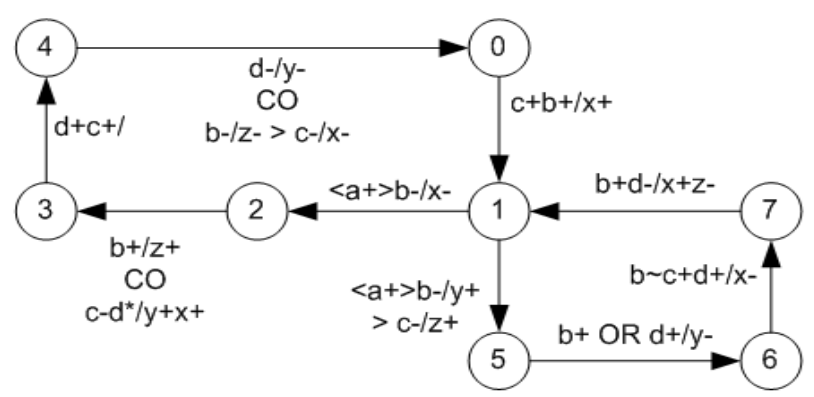

Figure 6. MBG specification

\section{GMBG Specification}

\subsection{I/O Sequence Behavior Using Burst Operators}

Consider the timing diagram shown in Figure $7 \mathrm{a}$

This behaviour can be described by both specification, STG and XBM. This behaviour may be described in XBM defining two sequential state transitions activated by the inputs bursts $(a+b * / x+y+)$ and $(b+c+/ z+)$ (Figure $7 \mathrm{~b})$. Suppose that the input burst $c+$ is activated immediately after the activation of the output signal $y+$ as a result there is a violat ion of the $\mathrm{I} / \mathrm{O}-\mathrm{Mode}$. The input/output $(\mathrm{I} / \mathrm{O})$ operation mode, says in which a new input is accepted as soon as an output transition has finished. If the 3D-tool synthesizes this behaviour, so delay elements should be inserted in the line of the signal $c$ to satisfy the $\mathrm{I} / \mathrm{O}-$ Mode. This procedure is not tailored for FPGAs. A more efficient solution consists of describing this behaviour through two sequential bursts, but activated immediately $\left(a+/ x^{1}+y^{2}+\right) \quad S E Q \quad\left(b^{1}+c^{2}+/ z+\right)$ exactly as the timing diagram showed in Figure 7a. This description creates a limited degree of concurrency between

\footnotetext{
3 State transitions without operator are of $\mathrm{T}_{\mathrm{s}}$ type.
} 
an input $c$ and the output $x$ (Figure 7c), therefore, it operates in the I/O-Mode. This example illustrates the extension of the SEQ operator.
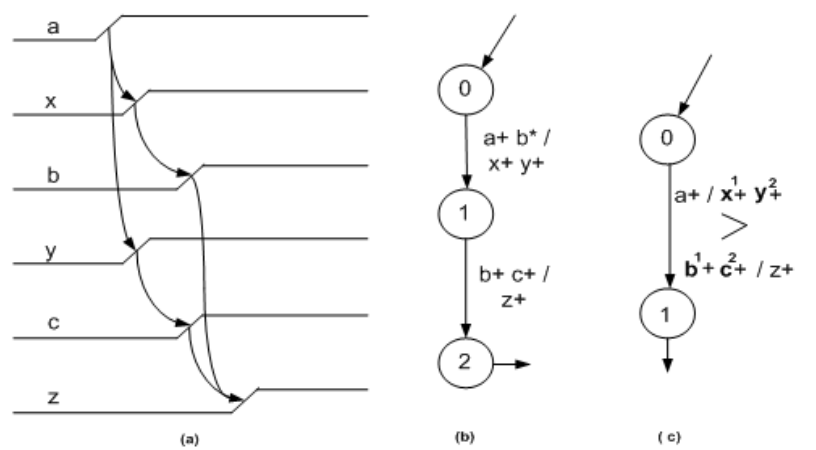

Figure 7. Specification types: a) timing diagram; b) XBM; c) GMBG (SEQ operator $\rightarrow$ symbol $>$ )

\subsection{Descriptions in GMBG}

Figure 8 shows some descriptions of benchmark controllers in GMBG that are originally described in STG, but on the other hand, these controllers cannot be described in XBM, because the reduction of concurrency between the signals of input / output is very high, invalidating the interaction with fast environments.

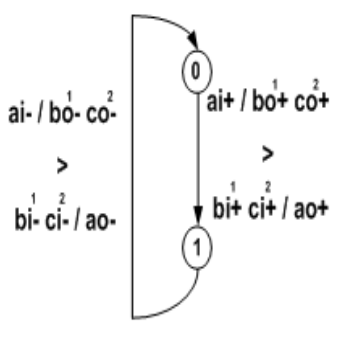

(a)

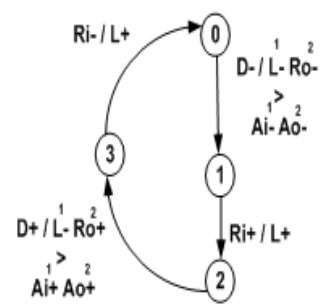

(b)

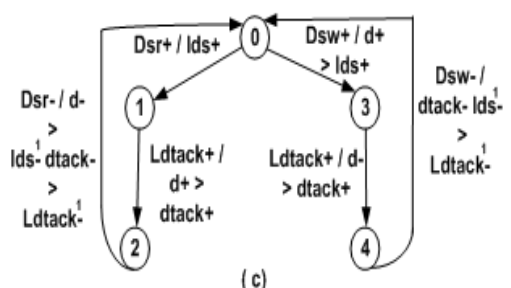

(c)

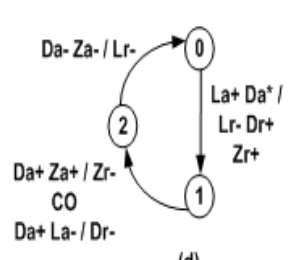

(d)
Figure 8. Benchmarks in GMBG: a) PAR; b) FIFO; c) VME bus; d) A/D Fast

\section{Synthesis Procedure}

Our controllers operate between state transitions in the GFM. In the state transitions with the SEQ operator, the controllers operate in $\mathrm{I} / \mathrm{O}$ mode. Our method begins from the GMBG specification and the procedure has six steps:

1. Behavioural capture using GMBG specification;

2. Transformation of the GMBG into a minimum set of

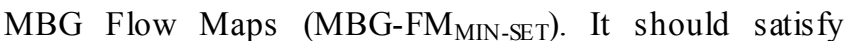
requirements that solve all conflicts and allow hazard-free logic minimization[10],[12] (see section A);
3. Codify the MBG-FM $M_{\text {MIN-SET }}$ avoiding critical race and with the lo west number of state variables[10];

4. Logic minimization (see section B) for each non-input signal $\left(\mathrm{F}_{\mathrm{SET}}\right.$ and $\left.\mathrm{F}_{\mathrm{RESET}}\right)$;

5. Technology mapping for each non-input signal $\left(\mathrm{F}_{\mathrm{SET}}\right.$ and $\left.\mathrm{F}_{\mathrm{RESET}}\right)[28]$;

6. Structural VHDL for each non-input signal $\left(\mathrm{F}_{\mathrm{SET}}\right.$ and $\mathrm{F}_{\text {RESET }}[30]$.

\subsection{Covered Conditions}

Figure 9 shows the MBG-FM (2 mean don't-care) involving state transition of the $\mathrm{T}_{\mathrm{SEQ}}$ type. The cells labelled with $\mathrm{R}$ in the figures are reserved and they aren't part of the possible paths (inputs bursts and outputs bursts activated), although they can be used in the logic cover. The set of reserved cells allows each non-input signal to hold a hazard-free logic cover with basic gates.

\begin{tabular}{|c|c|c|c|c|c|c|c|c|}
\hline$a^{a} b_{c}$ & 000 & 010 & 110 & 100 & 101 & 111 & 011 & 001 \\
\hline$y=000$ & ${ }^{0} 000$ & & $112^{R}$ & 110 & 112 & $112^{R}$ & & \\
\hline 010 & & & $112^{R}$ & 110 & 110 & $112^{R}$ & & \\
\hline 110 & & & 110 & 110 & 110 & 111 & & \\
\hline 100 & & & 110 & 110 & $112^{\circ}$ & $112^{\mathrm{R}}$ & & \\
\hline 101 & & & & & & & & \\
\hline 111 & & & & & & 221 1 & & \\
\hline 011 & & & & & & & & \\
\hline 001 & & & & & & & & \\
\hline
\end{tabular}

Figure 9. MBG Flow Map of Figure 7c

\subsection{Logic Hazard-Free Conditions}

For each non-input signal $z \in \mathrm{GMBG}$ that has a transition Tj $0 \rightarrow 1$ described in MBG flow map ${ }^{4}$, there is a cube that completely covers the paths of all cells of value 1 (minterms). To illustrate the concept of cube, extracted from the state transition $0 \rightarrow 1$ in Figure 9, the cube of the signal $x$ which is $\mathrm{C}_{\mathrm{TJ}-\mathrm{X}}(\mathrm{a}, \mathrm{b}, \mathrm{c}, \mathrm{x}, \mathrm{y}, \mathrm{z})=122220$, where 2 is don't-care. The theory of hazard-free logic minimization for XBM functions $\left(f_{G F M-X B M}\right)$ proposed by Nowick in[12] and Yun in [10] is extended to satisfy the Theorem 1 below, for the two-level function $f_{G M B G}\left(F_{S E T-G M B G}\right.$ and $\left.F_{R E S E T-G M B G}\right)$ of the feedback RS standard architecture and delay model is unbounded gate delay bounded wire delay (UGBWD).

Theorem 1: Two sufficient conditions for the implementation of hazard-free log ic circu its in the feedback RS standard architecture (see Figure 5) are:

The circuit doesn't have any reached state, which is covered for more than one cube (product).

The reachable states of the circuit that form the sequence of events $(0 \rightarrow 1 \ldots 1)$ or $(1 \rightarrow 0 \ldots 0)$ of a non-input signal should enable only one cube (product).

Proof: Let a sum-products function $f\left(\mathrm{~F}_{\mathrm{SET}-\mathrm{GMBG}}\right.$ and $\mathrm{F}_{\text {RESET-GMBG }}$ ) of a non-input signal $y \in \mathrm{GMBG}$ is hazard-free functional, that presents the transitions $\mathrm{T}_{\mathrm{J}}, \ldots, \mathrm{T}_{\mathrm{K}} 0 \rightarrow 1$ in the respective state transitions $J, \ldots, K$ and are described in the MBG flow table.

\footnotetext{
4 The treatment is similar to transition $1 \rightarrow 0$.
} 
Condition 1: Let the cubes (products) and $\mathrm{C}_{\mathrm{TJ}}$ and $\mathrm{C}_{\mathrm{TK}}$ of function $\mathrm{f}$ that completely cover all minterms (cells with value 1) of the respective transitions $\mathrm{T}_{\mathrm{J}}$ and $\mathrm{T}_{\mathrm{K}}$. If $\mathrm{C}_{\mathrm{Tj}} \cap$ $\mathrm{C}_{\mathrm{Tk}}=\varnothing$ then the cubes satisfy condition 1 . If $\mathrm{C}_{\mathrm{TJ}} \cap \mathrm{C}_{\mathrm{TK}} \neq \varnothing$ then there is at least one minterm that is covered by two cubes therefore violates condition 1 . If $\mathrm{C}_{\mathrm{TJ}} \subset \mathrm{C}_{\mathrm{TK}}$ then there is only the cube $\mathrm{C}_{\mathrm{TJ}}$, there fore satisfies the condition 1 .

Condition 2: If $\exists \mathrm{C}_{\mathrm{TJ}}$ a cube that completely covers the minterms of the transition $T_{J}$ ( $J$ state transition) and is adjacent to the $\mathrm{C}_{\mathrm{TK}}$ cube of the $K$ state transition, then $\mathrm{C}_{\mathrm{TJ}} \cup \mathrm{C}_{\mathrm{TK}}$ that generates a cube unique that satisfies the condition 2. Assuming that in the transitions $T_{\mathrm{J}}$ and $T_{K}$ there is the cubes $\mathrm{C}_{\mathrm{TJ}}$ and $\mathrm{C}_{\mathrm{TK}}$ not adjacent $\left(\mathrm{C}_{\mathrm{TJ}} \cap \mathrm{C}_{\mathrm{TK}}=\varnothing\right)$ and there is a path between the final states of the transitions of state $J$ to $K$, so this path the not-input signal is $1 \rightarrow 1$. As in the $J$ state transition the cube $\mathrm{C}_{\mathrm{TJ}}$ was activated and following the path until $K$, also the $\mathrm{C}_{\mathrm{TK}}$ cube is activated therefore violates condition 2 , because there are two cubes activated. As in the feedback RS standard architecture allows that the next transition following the $J$, in case the $\mathrm{C}_{\mathrm{TJ}}$ cube being disabled, so never occurs two cubes being activated, therefore satis fies the condition $2(c q d)$.

The theorem 1 shows that the $f_{G M B G}$ functions obtained are logic hazards-free for the specified multi-burst transitions types $\left\{T_{S}, T_{S E Q}, T_{C O}, T_{S-C}\right\}$. The logic cover of state transition of the type of the $T_{O R}$ of GMBG needs two cubes, so the delay model does not satisfy UGBWD. The theorem presented for the transition $0 \rightarrow 1$ is similar for the transition $1 \rightarrow 0$.

\section{Example}

In order to show our method, we used the Input D-Port Controller showed in the Figure 10a. Figure 10b show the version of this controller in GMBG specification (step-1). In this example is applied the extension of SEQ operator. The step-2 transforms the GMBG in two MBG-FM they are respected the requirements. The step-3 codifies the two MBG-Flow Maps using just one state variable ( $Y$ signal) (see Figure 11). Figures 12 and 13 show respectively the Karnaugh maps for the $R i$ signal $\left(\mathrm{R}_{\mathrm{i}-\mathrm{SET}}\right.$ and $\mathrm{R}_{\mathrm{i} \text {-RESET }}$ ) (step 4).

$$
\begin{gathered}
\mathrm{R}_{\mathrm{i}-\mathrm{SET}}=\text { Den.y'+Den'.y } \\
\mathrm{R}_{\mathrm{i} \text {-RESET }}=\text { Den y Rp' + Den' A i y' }
\end{gathered}
$$

The decomposition wasn't necessary (trivial technology mapping - step-5). Figure 13 shows the hazard-free logic circuit of the Input D-Port Controller synthesized by our method. The functions are described in structural VHDL (step-6).

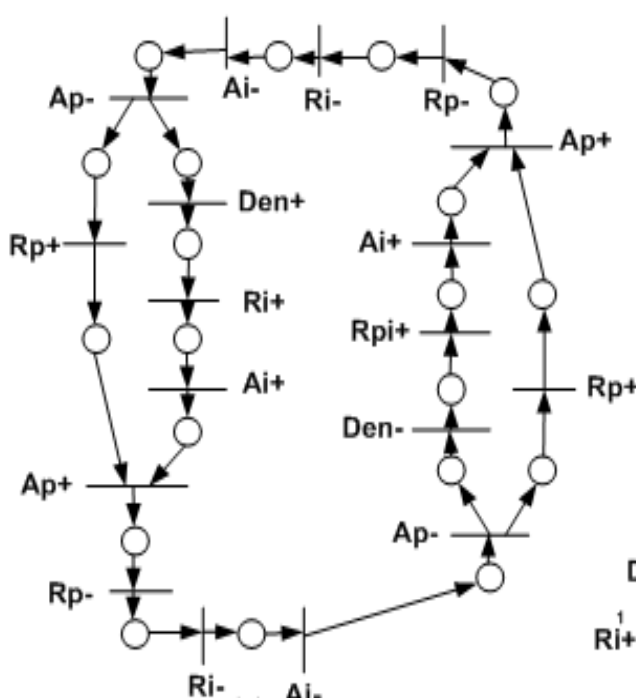

(a) Ai-

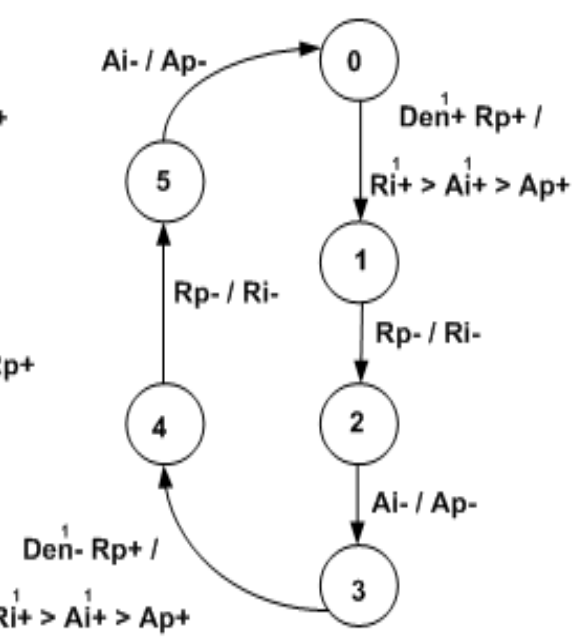

(b)

\begin{tabular}{|c|c|c|c|c|c|c|c|c|c|}
\hline \multirow{2}{*}{\multicolumn{2}{|c|}{ Ap Ri 00}} & 000 & 010 & 110 & 100 & 101 & 111 & 011 & 001 \\
\hline & & $000^{\circ}$ & 001 & & & & & 001 & 000 \\
\hline \multirow[t]{4}{*}{$Y=0$} & 01 & & 001 & 001 & & & 011 & 001 & \\
\hline & 11 & & & 111 & 010 & & $0111^{1}$ & & \\
\hline & 10 & 000 & & & 010 & & & & \\
\hline & 00 & 101 & $100\}^{3}$ & & & & & 100 & 101 \\
\hline \multirow[t]{3}{*}{$Y=1$} & 01 & 101 & & & 101 & 111 & & & 101 \\
\hline & 11 & & & 110 & 011 & $111{ }^{4}$ & & & \\
\hline & 10 & & 100 & 110 & & & & & \\
\hline
\end{tabular}

Figure 10. Specifications: a) ST G in[27]; b) GMBG version

Figure 11. MBG maps: minimized and codified 


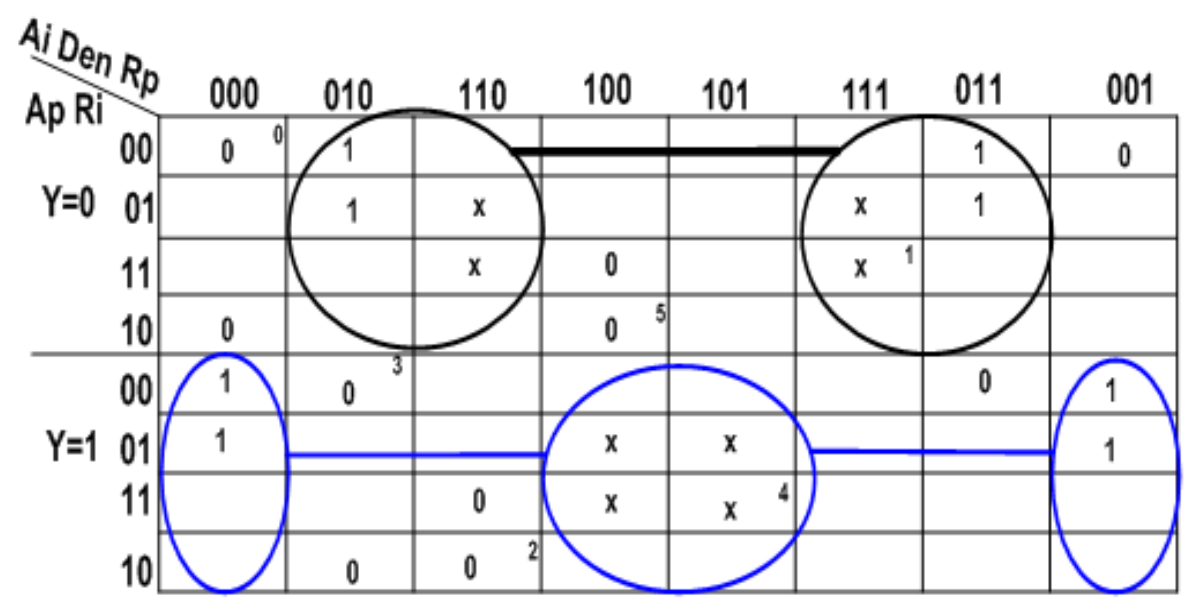

Figure 12. Karnaugh map: signal Ri-SET $\rightarrow$ function $F_{\text {SET }}$

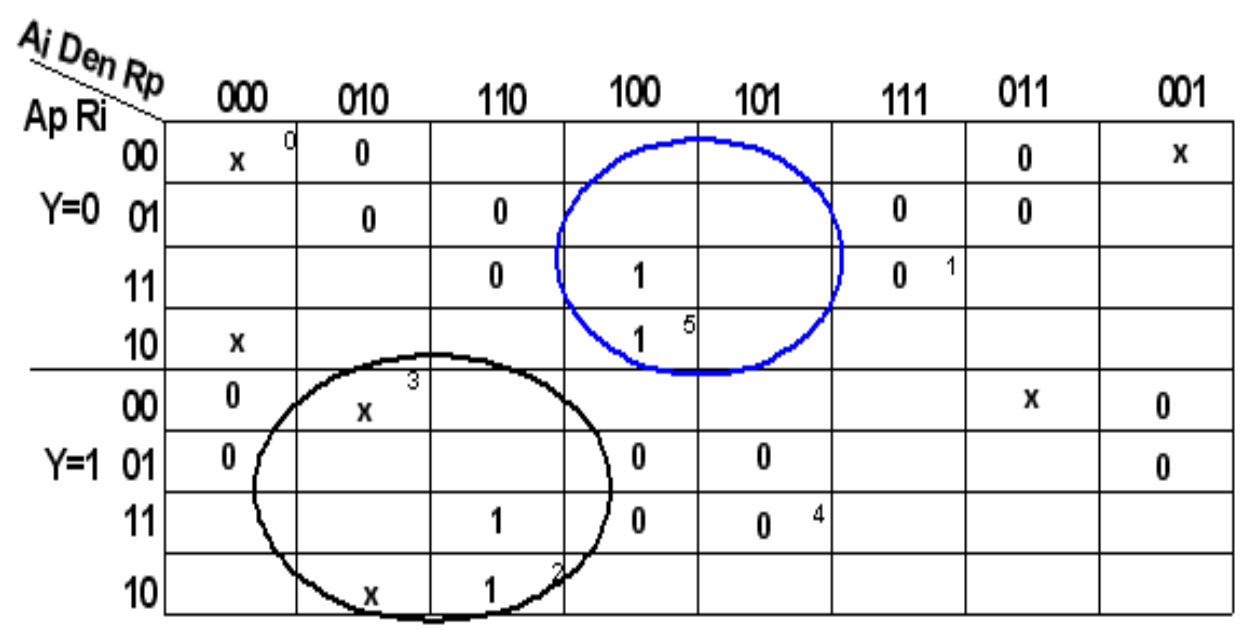

Figure 13. Karnaugh map: signal $\mathrm{Ri} \rightarrow$ function $\mathrm{F}_{\text {RESET }}$

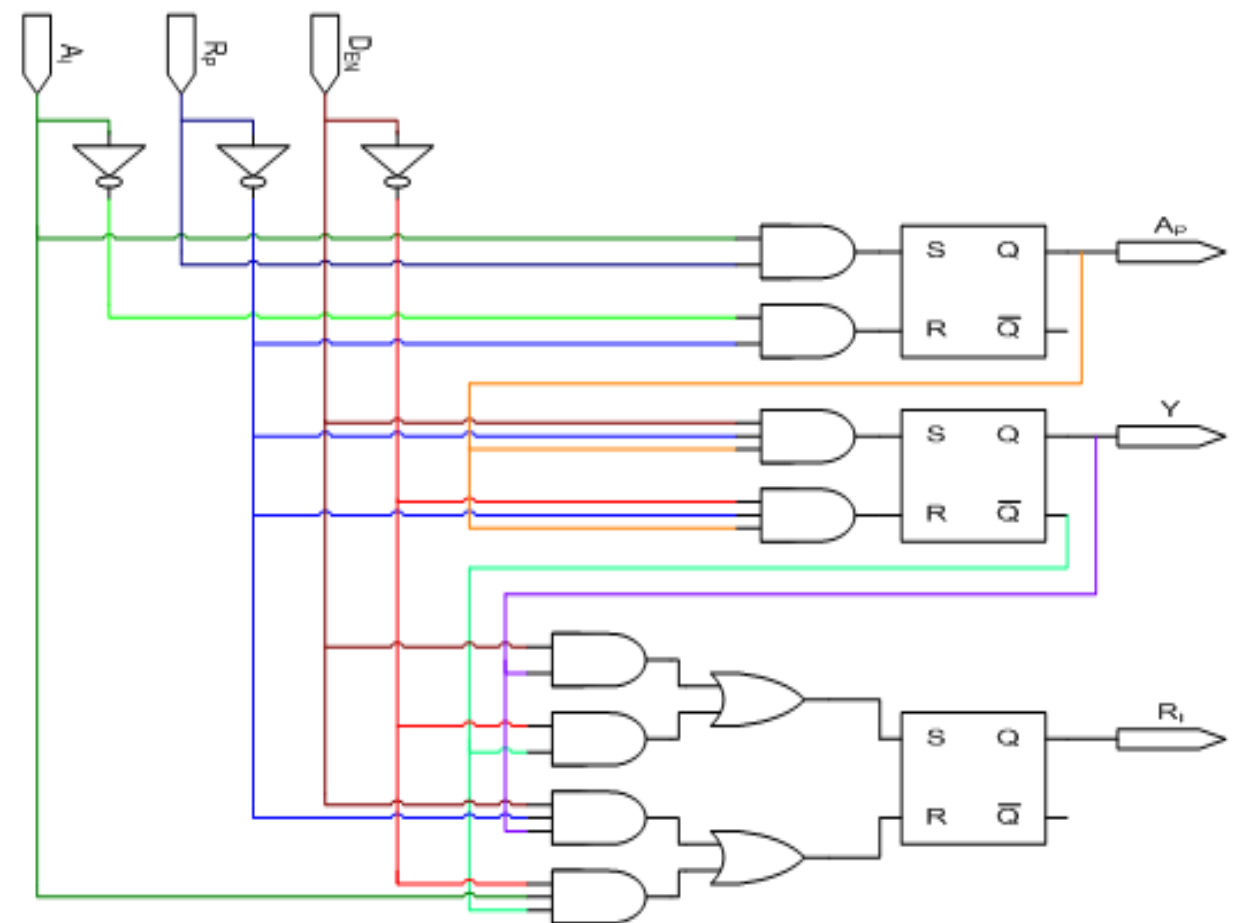

Figure 14. Logic Circuit: Input D-Port controller 


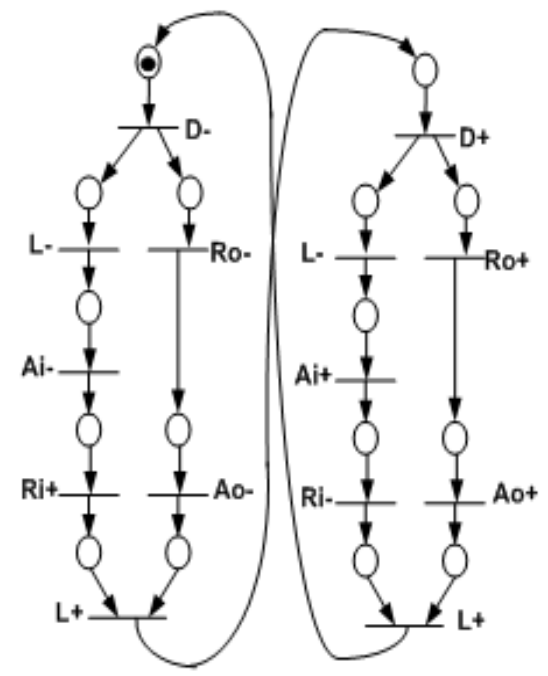

(a)

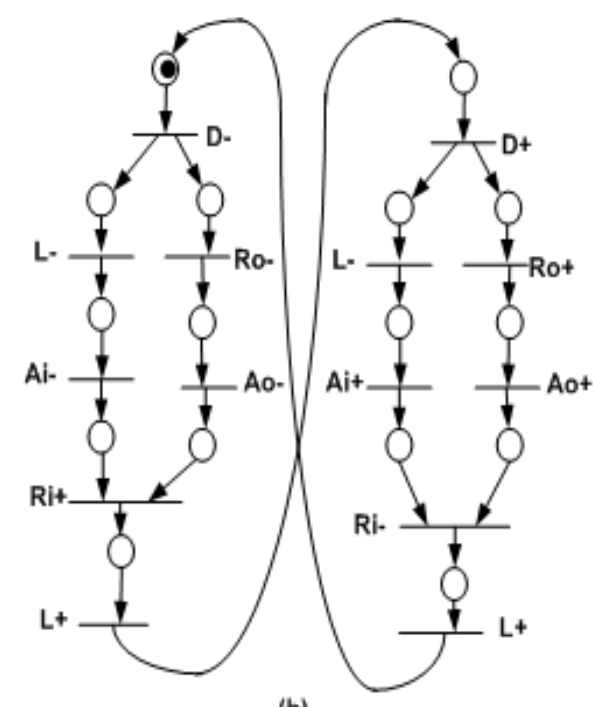

(b)

Figure 15. ST Gs of FIFO controller: a) original; b) reduced concurrency

\section{Results \& Discussion}

The strong of STG is to describe concurrency between inputs and outputs, important in the interaction with fast environments. The drawback is to describe decisions (input choices), by example, involving signals LSS, which are important in heterogeneous systems, where ST G can explode in the size, becoming very confusing.

The strong of XBM is to describe decisions involving signals LSS, concurrency of input signals and output signals. The drawback is to describe concurrency between signals of input and output, and sequence of signals.

The GMBG description is compact, not confused and it is familiar to designers of the synchronous digital c ircuit (based on the state diagram). The GMBG describes several types of concurrency, but still have some limitations. For example the GMBG description of the FIFO controller (Figure 8b) reduced the concurrence of the signal $R i$. Figure 15 a shows the original STG description of FIFO controller and Figure $15 \mathrm{~b}$ shows the equivalent FIFO controller depicted in the Figure 8b.

Through of timing diagram of a state transition, the Figure 16 shows the capacity of the GMBG in to describe concurrency and sequence. Beister et al. in[31] presents a procedure that transforms STG in XBM. The STG of the Figure 1 could be transformed in two $\mathrm{XBM}$ and just one GMBG (see Figure 4).

The Table-1 shows ten benchmarks that are described originally in STG and that were transformed in GMBG. The table contents the number of states and state transitions for the MBG transformation as well as the state variables, the number of LUTs and the maximum latency time for each benchmark. These circuits were simulated in the QUARTUS II to the target EP2C35F672C7[30]. The simulations didn't show any hazard problem and the circuit operated as predicted in its specification. Figure 17 shows the hazard-free waveforms extracted from the simulation of the Input D-Port Controller that the logic circuit is showed in Figure 14.

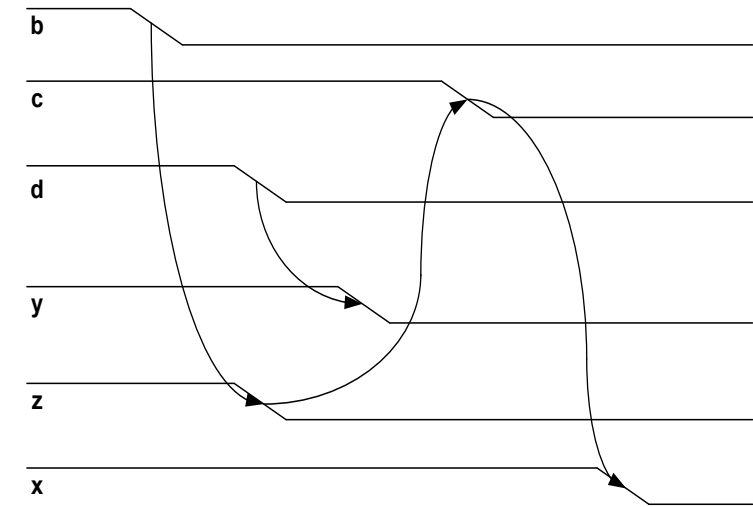

Figure 16. Timing diagram: state transition $4 \rightarrow 0$ of Figure 6

Table 1. Results: implement ation on FPGA

\begin{tabular}{|c|c|c|c|c|c|}
\cline { 2 - 6 } \multicolumn{1}{c|}{} & $\begin{array}{c}\text { States I } \\
\text { Transitions }\end{array}$ & $\begin{array}{c}\text { Inputs I } \\
\text { Outputs }\end{array}$ & $\begin{array}{c}\text { State } \\
\text { Variables }\end{array}$ & $\begin{array}{c}\text { Num ber } \\
\text { of LU T S }\end{array}$ & $\begin{array}{c}\text { Time } \\
\text { of Latency }\end{array}$ \\
\hline A.D/Fast & $3 / 3$ & $3 / 3$ & 0 & 7 & 10,987 \\
\hline A rbiter cell & $7 / 8$ & $3 / 3$ & 0 & 9 & 11,242 \\
\hline Converta & $4 / 4$ & $2 / 2$ & 1 & 9 & 9,908 \\
\hline D.Port [27] & $6 / 6$ & $3 / 2$ & 1 & 10 & 12,901 \\
\hline Fifo & $4 / 4$ & $3 / 3$ & 0 & 8 & 12,657 \\
\hline Figure 1 & $4 / 5$ & $3 / 3$ & 0 & 10 & 11,005 \\
\hline Par & $2 / 2$ & $3 / 3$ & 0 & 4 & 11,168 \\
\hline $\begin{array}{c}\text { Pipeline } \\
\text { Handshaking }\end{array}$ & $2 / 2$ & $2 / 2$ & 0 & 4 & 10,926 \\
\hline \begin{tabular}{c} 
VME bus \\
\hline W P Port
\end{tabular} & $5 / 6$ & $3 / 3$ & 0 & 8 & 12,914 \\
\hline
\end{tabular}




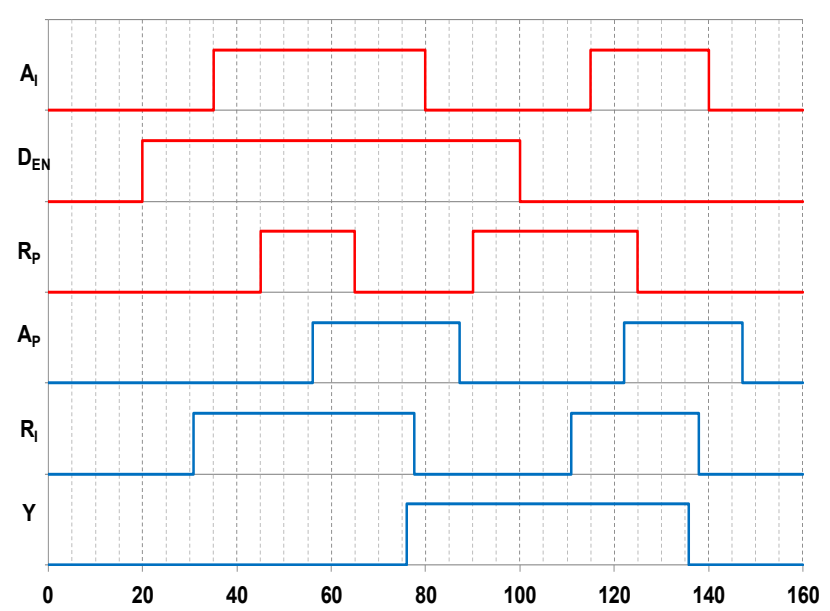

Figure 17. Timing diagram: input D-Port of Figure 10b

\section{Conclusions}

This paper shows the MBG specification. It describes all signals types of the XBM specification and I/O concurrence with CO and SEQ operators. The extension SEQ operator allows the circuit interacting with the environment in the $\mathrm{I} / \mathrm{O}$ mode. We also show that multi-burst mode controllers can be implemented on LUT-based FPGA in the BGWD class and using RS latches. For future work we intend to develop a tool for automatic synthesis. And we intend to go further on large asynchronous controllers in FPGA.

\section{REFERENCES}

[1] S. Hauck, "Asynchronous Design Methodologies: An Overview," in Proc. of the IEEE, Vol. $83: 1$ pp.69-93, January 1995.

[2] C. J. Myers, "Asynchronous Circuit Design," Wiley \& Sons, Inc., 2004, 2 a edition.

[3] S. Kim and P. A. Beerel, "Pipeline Optimization for Asynchronous Circuits: Complexity analysis and an Efficient Optimal Algorithm," IEEE Trans. CAD of Integrated Circuits and Systems, vol.25, no. 3, pp.389-402, March 2006.

[4] A. L. Plana and S. M. Nowick, "Architectural Optimization for Low-Power Nonp ip elined Asynchronous sy stems," IEEE Trans. on VLSI Systems, vol.6 no.1, pp.56-65, March 1998.

[5] T. Chelcea, et al., "A Burst-Mode Oriented Back-end for the Balsa Synthesis System," in Proc. Design, Automation and Test in Europe (DATE), pp.330-337, March 2002.

[6] L. Lavagno, "Synthesis and Testing of Bounded wire delay asynchronous circuits from signal transition graphs," Ph.D. dissertation, University California, Berkeley, 1992.

[7] T. -A. Chu, "Synthesis of Self-Timed VLSI Circuits from Graph-Theoretic Specifications," PhD thesis, June, Dept. of EECS, MIT, 1987.
[8] Yakovlev, A. V., "On Limitations and Extensions of STG model for Designing Asynchronous Control Circuits," in Proc. Int. Conf. Computer Design, pp. 396-400, October, 1992

[9] E. Pastor, J. Cortadella, A. Kondratyev and O. Roig, "Structural methods for the synthesis of speed-independent circuits," IEEE Trans. CAD, vol 17, pp. 1108-1129, November, 1998.

[10] K. Y. Yun and D. L. Dill, “Automatic Synthesis of Extended Burst-Mode Circuits: Part I (Specification and Hazard-Free Implementation) and Part II (Automatic Synthesis)," IEEE Trans. on CAD of Integrated Circuit and Systems, Vol. 18:2, February, pp. 101-132, 1999.

[11] A. L. Davis, "The architecture and system method for DDM 1: A recursively structured data-drive machine," In 5th Annual Symposium on Computer Architecture, April 1978.

[12] S. M. Nowick, "Automatic Synthesis of Burst-Mode Asynchronous Controllers," PhD thesis, Stanford University, 1993.

[13] K. Y. Yun and D. L. Dill, "A High-Performance Asynchronous SCSI Controller," in Proc. Int. Conf. Computer Design (ICCD), pp. 44-49, 1995.

[14] K. Y. Yun, et al. "The design and verification of a high-performance low-control overhead asynchronous differential equation solver," IEEE Trans. on VLSI Systems, vol.6, no4, pp. 643-655, December 1998.

[15] K. S. Stevens, et al., "An Asynchronous Instruction Length Decoder," IEEE Journal of Solid-state Circuits, vol. 36, no. 2, pp.217-228, February 2001.

[16] S. H. Unger, "Asynchronous Sequential Switching Circuits," John Wiley \& Sons Inc, 1969.

[17] P. Vanbekbergen, et al. "A generalized signal transition graph model for specification of complex interfaces," European Design and Test Conference, EDAC, pp.378-384, March, 1994.

[18] O. Kraus and M. Pedeffke, "XBM2PLA: A Flexible Synthesis Tool for Extended Burst Mode Machines," in Proc. Of the Design Automation and Test in Europe Conference and Exhibition, pp.1301-1303, 2003.

[19] O. Kraus, "Synthese von digitalen Asynchronen Zustandsautomaten," thesis - Doktor-Ingenieur Universitat Erlan gen-Nurnberg, 2003.

[20] D. L. Oliveira, M. Strum, W. J. Chau and W. C. Cunha, M iriã: a CAD toll synthesize multi-burst controllers for heterogeneous systems, Microelectronics Reliability, 43 (209-213), 2003

[21] D. L. Oliveira, "Miriã: uma ferramenta para síntese de controladores assíncronos multi-rajada,", Tese de Doutorado, Universidade de São Paulo (in Portuguese), 2004.

[22] D. L. Oliveira, et al., "Synthesis Concurrent Asynchronous State Machine using Extended Multi-Burst Graph Specification,” XIII Workshop Iberchip, Lima, Perú, 2007.

[23] Brunvand, E., "Using FPGAs to Implement Self-Timed Systems", Journal of VLSI Signal Processing, Special issue on field programmable logic, vol.6(2), pp.173-190, August 1993. 
[24] Maheswaran, K. and Akella, Venkatesh, "Hazard-Free Implementation of the Self-Timed Cell Set in a Xilinx FPGA.," Technical report, U.C. Davis, 1994.

[25] R. Payne, “Asynchronous FPGA architectures," IEE Proc. Comp. Digit. Tech., vol.143, no.5, pp.282-286, September, 1996.

[26] Muttersbach, J., Villiger, T. and Fichtner, W., "Practical Design of Globally-Asynchronous Locally-Synchronous Systems," in Proc. Sixth Int. Symposium on Advanced research in Asynchronous Circuits and systems, pp.52-59, April 2000.

[27] Najibi, M. et al., "Prototyping Globally Asynchronous Locally Synchronous Circuits on Commercial Synchronous
FPGA," The 6th IEEE Int. Workshop on Rapid system Prototyping, pp.63-69, 2005

[28] Sigel, P., De Michele, G. and Dill, D., "Decomposition methods for library binding of speed-independent," Proc. Int. Conf. Computer-Aided Design, pp.558-565, November 1994.

[29] D. L. Oliveira, et al., "Automatic Synthesis of Essential Hazard-Free Burst-Mode Asynchronous circuit," in Proc. ANDESCON, Perú, Cusco, 2008.

[30] Altera Corporation, www.altera.com.

[31] J. Beister, et al., "From STG to Extended-Burst-Mode Machines," in Proc. Fifth Int. Symposium on Advanced Research in Asynchronous Circuits and Systems,pp.145-156, 1999. 